\title{
Repelência e toxicidade de Cyperus iria L., em início de florescimento, ao gorgulho Sitophilus oryzae
}

\author{
$\overline{\text { Ana L. de A. P. Capps }{ }^{1} \text {, José P. S. Novo }{ }^{2} \& \text { Maria do C. de S. S. Novo }}$
}

\section{RESUMO}

Visa-se, com este trabalho, avaliar a repelência e a toxicidade de Cyperus iria L., no estádio de início de florescimento, na Sitophilus oryzae (L.). Realizaram-se dois bioensaios, avaliando-se pós de raízes, partes aéreas e inflorescências de C. iria misturados a grãos de trigo, nas concentrações de 0, 1, 2 e 5\%. Em um terceiro bioensaio, avaliou-se a repelência de extratos aquosos produzidos a partir dos pós de C. iria impregnando-se papel sulfite nas concentrações de $0,0,1$, 0,5 e $1,0 \mathrm{mg}$ de planta $\mathrm{cm}^{-2}$. Os pós produzidos com qualquer uma das estruturas de C. iria, repeliram a S. oryzae. A repelência e a toxicidade foram dependentes da concentração e da estrutura da planta empregada. A repelência foi maior com $5 \%$ de raízes e menor quando se usou qualquer uma das concentrações da inflorescência e se manteve, em raízes, por períodos superiores a 150 dias. A mortalidade e a redução na progênie foram maiores quando empregados pós de raízes de C. iria, exceto com $2 \%$ de raiz, todas as outras estruturas reduziram a progênie de $S$. oryzae até 60 dias. Houve repelência quando empregados extratos aquosos de raízes a 0,1 e $0,5 \mathrm{mg} \mathrm{cm}^{-2}$, de parte aérea $0,5 \mathrm{mg} \mathrm{cm}^{-2}$ e de inflorescência a 0,5 e $1,0 \mathrm{mg} \mathrm{cm}^{-2}$. O tratamento de grãos com pós de $C$. iria foi mais eficiente no controle de $S$. oryzae que os extratos aplicados em papel.

Palavras-chave: insecta, biologia, inibição de crescimento, análogos de hormônio juvenil

\section{Repellence and toxicity of Cyperus iria L., at beginning of flowering, to rice weevil Sitophilus oryzae}

\begin{abstract}
The aim of this work was to evaluate the repellence and toxicity of Cyperus iria L., at beginning of flowering, to Sitophilus oryzae (L.). Two bioassays were used to evaluate the powders produced from the roots, shoots and inflorescence of $C$. iria, mixed with wheat grains in concentrations of $0,1,2$ and $5 \%$. A third bioassay evaluated the repellence of the aqueous extracts produced from the powders of $C$. iria impregnated in paper in concentrations of 0 , $0.1,0.5$ and $1.0 \mathrm{mg}$ of plant $\mathrm{cm}^{-2}$. The powders produced with any structure of $C$. iria repelled S. oryzae. The repellence and toxicity depended on concentration and plant structure used in the production of the powder. The repellence was higher with $5 \%$ of root and lower with any other concentration of inflorescence. With roots, it was present for periods longer than 150 days. The mortality and reduction in progeny were higher when the roots of $C$. iria were tested. All treatments reduced the progeny of $S$. oryzae for up to 60 days, with the exception of the concentration of $2 \%$ of the root. When aqueous extracts from the structures of $C$. iria were employed, repellence was verified for roots at 0.1 and $0.5 \mathrm{mg} \mathrm{cm}^{-2}$, for aerial parts at $0.5 \mathrm{mg} \mathrm{cm}^{-2}$, and flowers at 0.5 and $1.0 \mathrm{mg} \mathrm{cm}^{-2}$. The treatment of grains using powders from $C$. iria was more efficient in the control of $S$. oryzae than the extracts applied on paper.
\end{abstract}

Key words: insecta, biology, growth inhibition, juvenile hormone analogues

\footnotetext{
1 Estudante de Iniciação Científica, Bolsista PIBIC/CNPq, Centro de Pesquisa e Desenvolvimento de Fitossanidade, Instituto Agronômico (IAC), CEP 13001-970, Campinas, SP. Fone: (19) 3241-5188, ramal 359. E-mail: analucapps@yahoo.com.br

2 Pesquisador Científico, Centro de Pesquisa e Desenvolvimento de Fitossanidade, IAC, CP 28, CEP 13001-970, Campinas, SP.

3 Pesquisador Científico, Centro de Pesquisa e Desenvolvimento de Ecofisiologia e Biofísica, IAC, CP 28. Fone: (19) 3241-5188, ramal 430. E-mail: jpsnovo@iac.sp.gov.br
} 


\section{INTRODUÇÃO}

Anualmente, a perda de grãos na pós-colheita, decorrente da ação de insetos, de deterioração microbiana e de outros fatores, é estimada em 10 a $25 \%$ da produção mundial (Mohan \& Fields, 2002). No Brasil, os gorgulhos são considerados as pragas mais importantes de grãos armazenados, por apresentarem alto potencial biótico, infestação cruzada, capacidade de penetração nas massas de grãos, elevado número de hospedeiros e também pelo fato de que tanto suas larvas como os adultos danificam os grãos (Gallo et al., 2002). O gorgulho Sitophilus oryzae (L.) (Coleoptera: Curculionidae) é um inseto cosmopolita encontrado em todas as regiões quentes e tropicais causando danos a grãos de arroz, cevada, milho, trigo, sorgo e em cereais processados como o macarrão (Metcalf \& Flint, 1962). Contamina ainda os grãos com suas exúvias e excrementos, aumenta o nível de ácidos graxos livres, contaminando-os com ácido úrico, causando odor desagradável, reduzindo o rendimento e a qualidade das farinhas (Boff et al., 2005).

A aplicação sistemática e contínua de inseticidas líquidos e gasosos tem sido o principal método de controle do gorgulho mas, com o uso constante, indiscriminado e incorreto, houve diminuição de sua eficiência, sendo necessário aumentar o número de aplicações, propiciando o surgimento de populações resistentes e elevando o custo da aplicação (Bogorni \& Vendramin, 2003). Como consequência ocorreu aumento no número de casos de intoxicação nos aplicadores e se observou efeito nocivo em organismos não alvo no ambiente e a presença de resíduos nos alimentos (Coitinho et al., 2006). A necessidade de se encontrar novas moléculas menos tóxicas e com menor impacto ambiental é de primordial importância, o que tem estimulado e aumentado o interesse de pesquisa com plantas inseticidas (Pungitore et al., 2005).

Entre os muitos metabólitos secundários produzidos por plantas que interferem nas funções fisiológicas dos insetos, estão os análogos de hormônio juvenil; são sesquiterpenóides que regulam processos fisiológicos críticos, como a metamorfose e a reprodução (Edwards et al., 1991), sintetizados pelo órgão endócrino retrocerebral, a corpora allata. De acordo com Bede et al. (2001), o hormônio juvenil III (HJ III) e seu precursor, metil-farnesoato (MF), ativos no controle de algumas espécies, foram identificados em junquinho, Cyperus iria L., que é uma espécie daninha pertencente à família Cyperaceae, que infesta principalmente lavoura de arroz de várzea ou irrigada, competindo na fase inicial do crescimento da cultura (Lorenzi, 2006).

Existem poucos trabalhos sobre a interação entre $C$. iria e insetos; entretanto, em um experimento realizado na Malásia, Toong et al. (1988) isolaram, de plantas de C. iria, um composto semelhante ao HJ III, com efeito morfogenético em vários insetos. O gafanhoto Melanoplus sanguinipes (Fabricius, 1789) (Orthoptera: Acrididae), criado em laboratório empregando-se o C. iria como hospedeiro apresentou na fase adulta, 90\% dos indivíduos com deformações nas asas. Na presença de excesso de hormônio juvenil observaram-se mudanças cromáticas características do desenvolvimento com as fêmeas apresentando ovários com oócitos imaturos e ovos poucos desenvolvidos que, posteriormente, se tornaram estéreis.

Em condições de campo, Meneses \& Garcia de la Osa (1988) observaram oviposição de Hydrellia sp (Diptera: Ephydridae) em folhas de C. iria mas seus ovos não eram viáveis. Constatou-se, em avaliação de preferência alimentar, que o percevejo Oebalus pugnax (Fabricius, 1775) (Hemiptera: Pentatomidae), se alimentou de $C$. iria, embora um número maior de indivíduos tenha preferido a poácea Paspalum urvillei Stend.

Novo et al. (2003) avaliaram o efeito de C. iria no desenvolvimento de $S$. oryzae, quando adicionada a pellets produzidos com farinha de trigo integral e verificaram que houve ação inseticida dependente da concentração; no tratamento com $10 \%$ de C. iria, a porcentagem de inibição do crescimento foi de $98,85 \%$. Segundo os autores, o prolongamento na duração da fase ninfal e/ou pupal, assim como o efeito na viabilidade e nas fases do inseto, pode ser atribuído aos metabólitos secundários existentes em $C$. iria, entre eles o HJ III.

As substâncias vegetais com ação inseticida podem ser empregadas na forma de pós, extratos e óleos (Boff et al., 2006). A mistura direta de extrato ou de pós de plantas aos grãos armazenados é uma das formas mais simples de aplicação, sendo especialmente indicada para a agricultura de subsistência devido ao seu baixo custo, facilidade de uso, menor risco aos aplicadores e consumidores. Diversas plantas têm sido testadas quanto ao seu potencial inseticida a grãos armazenados. Dales (1996) relacionou mais de 120 espécies com efeito inseticida, repelentes ou deterrente. Em estudo sobre a preferência alimentar de $S$. oryzae, observouse que grãos de trigo tratados com extratos de sementes da apiácea Anethum graveolens L. (dill), atraíram menos insetos que a testemunha (Su, 1985); esta mesma autora, utilizando tiras de papel tratadas ou não com $0,68 \mathrm{mg} \mathrm{cm}^{-2}$ do extrato de $A$. graveolens diluído em acetona, verificou que o efeito repelente se manteve por até 60 dias.

Há falta de informações sobre o efeito da mistura direta de pós e de extratos de $C$. iria aos grãos de trigo visando ao controle de $S$. oryzae. Objetivou-se com o trabalho avaliar os efeitos tóxicos e a repelência de pós e de extratos de plantas de $C$. iria, colhidas no estádio de início de florescimento e adicionados a grãos de trigo e a tiras de papel, além de determinar o seu efeito residual.

\section{MATERIAL E MÉTODOS}

Solo de várzea do Centro Experimental Central do Instituto Agronômico (IAC), em Campinas, SP, foi retirado da camada 0-20 cm, secado à sombra e peneirado para a retirada de torrões e de restos vegetais. Os resultados das análises químicas de amostras coletadas na área do experimento foram: P: $46 \mathrm{mg} \mathrm{dm}^{-3}$; matéria orgânica: $65 \mathrm{~g} \mathrm{dm}^{-3}$; $\mathrm{pH}$ em $\mathrm{CaCl}_{2:}$ 4,3; K: 0,6 $\mathrm{mmol}_{\mathrm{C}} \mathrm{dm}^{-3}$; Ca: $17 \mathrm{mmol}_{\mathrm{C}} \mathrm{dm}^{-3}$; $\mathrm{Mg}$ : $7 \mathrm{mmol}_{\mathrm{C}} \mathrm{dm}^{-3}$; H+Al: $150 \mathrm{mmol}_{\mathrm{C}} \mathrm{dm}^{-3}$; SB: 24,6 $\mathrm{mmol}_{\mathrm{C}} \mathrm{dm}^{-3}$ CTC: $174,2 \mathrm{mmol}_{\mathrm{C}} \mathrm{dm}^{-3} ; \mathrm{V}: 14 \%$. A terra foi colocada em vasos plásticos de $16 \mathrm{~cm}$ de altura e diâmetros superior e 
inferior, respectivamente, de 18 a $12,5 \mathrm{~cm}$ e perfurados para percolação da solução do solo. A terra não foi corrigida nem adubada.

Dissemínulos de C. iria provenientes da Estação Agrícola Experimental da Bayer CropScience, Paulínia, SP, foram semeados em 5/6/2003, na razão de $1 \mathrm{~g}$ de sementes por vaso. Após a semeadura os vasos foram irrigados e, em seguida à germinação, que ocorreu de 9/6/2003, mantidos inundados até a colheita final. No início do florescimento as plantas foram cortadas rente ao solo, e as raízes, partes aéreas e inflorescência, separadas, lavadas retirando-se o excesso de água com papel absorvente. Todas as estruturas foram secadas em estufa sob ventilação forçada, a $30-38^{\circ} \mathrm{C}$ até peso constante. As estruturas, após a secagem, foram moídas em moinho tipo Wiley, peneiradas sob crivo de 0,25 mm e mantidas em frascos de vidro, tampados até a instalação dos experimentos.

A criação de $S$. oryzae foi mantida em sala climatizada (temperatura de $25 \pm 2{ }^{\circ} \mathrm{C}$, umidade relativa de $70 \pm 10 \%$ e fotofase de $12 \mathrm{~h}$ ), no Laboratório de Entomologia do Centro de Pesquisa e Desenvolvimento de Fitossanidade (IAC), em frascos de vidro de $600 \mathrm{~mL}$, com tampas teladas, contendo grãos de trigo (Triticum aestivum L. cv IAC-60). Os grãos de trigo utilizados para a criação de insetos e nos experimentos, antes de serem usados, foram condicionados em garrafas tipo PET e mantidos em freezer doméstico durante sete dias para eliminação de eventuais infestações; por fim, três bioensaios foram realizados.

\section{Bioensaio 1: Avaliação da repelência de pós produzidos com as diferentes estruturas de $C$. iria a $S$. oryzae}

Os pós de raízes, partes aéreas e inflorescências de $C$. iria foram misturados aos grãos de trigo, nas concentrações de 1 , 2 e $5 \%$, e homogeneizados mecanicamente durante um minuto, de maneira a possibilitar distribuição uniforme do produto sobre os grãos; em um recipiente circular de vidro de $25 \mathrm{~cm}$ de diâmetro interno, colocou-se uma arena de isopor de mesmo diâmetro, com 10 furos de $3 \mathrm{~cm}$ de diâmetro; em cada furo havia um recipiente plástico com $3 \mathrm{~cm}$ de diâmetro por $1,5 \mathrm{~cm}$ de profundidade no qual foram colocados $5,75 \pm 0,4 \mathrm{~g}$ de trigo misturado com as estruturas de $C$. iria nas concentrações acima descritas e mais um tratamento testemunha, composto apenas de grãos de trigo; dentro de cada arena os tratamentos foram dispostos inteiramente ao acaso.

Liberaram-se, no centro da arena, 100 adultos não sexados de S. oryzae, com cinco dias de idade; após 24 h, contou-se o número de insetos em cada tratamento. Os dados foram convertidos para expressar a porcentagem de repelência empregando-se a fórmula de Talukder \& Howse (1994).

O bioensaio foi realizado no dia do tratamento dos grãos e repetido a cada trinta dias, até 150 dias; realizaram-se, em cada época de avaliação, dez repetições para cada tratamento. Para verificar se havia homocedasticidade da variância empregou-se o teste de Hartley (Gomes \& Garcia, 2002), e como se observou heterogeneidade da variância, transformaram-se os dados de número de insetos em $\sqrt{x}$ e os de porcentagem de repelência em arc sen $\sqrt{x / 100}$. Os valores médios das porcentagens de repelência foram clas- sificados de acordo com a escala preconizada por Talukder \& Howse (1994).

Realizou-se a análise de variância dos resultados de acordo com o método para experimentos em parcelas subdivididas (Gomes \& Garcia, 2002) e, quando significativos, os efeitos dos tratamentos com as diferentes partes da planta de C. iria, tiveram suas médias comparadas pelo teste de Duncan a 5\% de probabilidade; para avaliar o efeito residual dos pós realizaram-se análises de regressão polinomial.

\section{Bioensaio 2: Avaliação da toxicidade e do efeito na progênie de $S$. oryzae}

A cultivar de trigo empregada, assim como a preparação dos grãos desse bioensaio, foi a mesma utilizada no experimento anterior. Em placas de Petri plásticas, com $7 \mathrm{~cm}$ de diâmetro, foram postos $6 \mathrm{~g}$ de grão de trigo que já haviam sido misturados a pós de raízes, partes aéreas e inflorescências de $C$. iria, nas concentrações de 1,2 e 5\%; havia, também, um tratamento testemunha com apenas grãos de trigo. Logo após os tratamentos dos grãos e aos 30, 60, 90 e 120 dias depois, cada placa foi infestada com dez adultos de $S$. oryzae, não sexados e com idade média de cinco dias. Os insetos foram mantidos durante dez dias em contato com o material e depois retirados, avaliando-se a mortalidade; para cada tratamento havia cinco repetições.

Quanto à avaliação da progênie, os tratamentos se dispuseram na sala de criação, inteiramente ao acaso, com cinco repetições, durante 70 dias, sendo o número de insetos emergidos avaliados a cada 10 dias, a partir do $40^{\circ}$ dia. A redução ocorrida na progênie devido à ação dos tratamentos (PR) em relação à testemunha, foi calculada através da equação de Abbott (1925):

$$
\mathrm{PR}=((\mathrm{TEST}-\mathrm{TRAT}) / \mathrm{TEST}) * 100
$$

em que TEST: número de insetos no tratamento testemunha e TRAT número de insetos no tratamento com $C$. iria.

Devido à heterogeneidade da variância, os dados de porcentagem de mortalidade foram transformados em arc sen $\sqrt{x / 100}$ e os de progênie, em $\sqrt{x+1}$. Realizou-se a análise de variância dos resultados de acordo com o método para experimentos em parcelas subdivididas (Steel \& Torre, 1981) e, quando significativos, os efeitos dos tratamentos das diferentes partes da planta de $C$. iria tiveram suas médias comparadas pelo teste de Duncan a 5\% de probabilidade; para o efeito do residual das estruturas de $C$. iria nos grãos, empregou-se a análise de regressão polinomial e se escolheu a equação de regressão representativa de cada tratamento; consideraram-se a lógica do fenômeno biológico, a significância da análise de variância da regressão e o valor do coeficiente de determinação.

\section{Bioensaio 3: Avaliação de repelência de extratos aquosos em tiras de papel}

Os extratos foram preparados agitando-se mecanicamente os pós das diferentes estruturas de C. iria com $60 \mathrm{~mL}$ de água destilada e filtrados em papel de filtro Whatman $n^{0} 1$; os extratos aquosos de parte aérea, raízes e inflorescências 
foram aplicados em papel sulfite A5 cortados em pedaços de 17 x $17 \mathrm{~cm}$, nas doses de 0, 0,1, 0,5 e 1,0 mg de planta $\mathrm{cm}^{-2}$; o controle recebeu apenas água destilada. Antes de serem utilizados os papéis foram secados ao ar.

O papel sulfite foi cortado ao meio, e se uniram as metades tratadas e não tratadas; sobre os papéis se puseram anéis de vidro de $17 \mathrm{~cm}$ de diâmetro e $5 \mathrm{~cm}$ de altura, ficando metade da arena com papel tratado e a outra metade com o não tratado. No centro de cada arena foram liberados 20 adultos de $S$. oryzae não sexados, com cinco dias de idade e colocados no escuro; diariamente, no dia e 30 dias depois do tratamento e após uma hora, se avaliou o número de insetos em cada tratamento.

O delineamento experimental foi inteiramente ao acaso com dez repetições para cada tratamento; as porcentagens de repelência foram calculadas pela fórmula de Talukder \& Howse (1994) e, devido à heterogeneidade da variância, transformaram-se os dados em arcsen (x/100) 0,5 submetendo-os à análise de variância; enfim, compararam-se as médias pelo teste $\mathrm{t}$ a $5 \%$ de probabilidade.

\section{RESULTADOS E DISCUSSÃO}

\section{Bioensaio 1: Avaliação da repelência de pós produzidos com as diferentes estruturas de $C$. iria a $S$. oryzae}

Os resultados das avaliações de repelência de grãos de trigo tratados com pós produzidos com estruturas de $C$. iria sobre adultos de $S$. oryzae, são apresentados nas Tabelas 1 e 2; até 150 dias após os tratamentos todas as estruturas de $C$. iria repeliram S. oryzae (Tabela 1) mas esta foi dependente da concentração e da estrutura da planta; os tratamentos produzidos com pós de inflorescências e de raízes foram, respectivamente, os menos e os mais repelentes, em que a repelência foi maior no tratamento com $5 \%$ de pó de raízes. Bede \& Tobe (2000) observaram mudanças temporais e espaciais nos níveis de HJ III durante o desenvolvimento de C. iria, e que os maiores níveis de HJ III estavam localiza-

Tabela 1. Número médio de adultos de $S$. oryzae observados nos tratamentos em que grãos de trigo foram misturados com pós as estruturas raiz, parte aérea e inflorescência (infloresc.) de C. iria, nas avaliações realizadas logo após a mistura e aos 30,60, 90, 120 e 150 dias depois

\begin{tabular}{lcccccc}
\hline & \multicolumn{5}{c}{ Número médio de insetos adultos $^{1}$} \\
\cline { 2 - 7 } Tratamento após os tratamentos & \multicolumn{5}{c}{} \\
\cline { 2 - 7 } & $\mathbf{0}$ & $\mathbf{3 0}$ & $\mathbf{6 0}$ & $\mathbf{9 0}$ & $\mathbf{1 2 0}$ & $\mathbf{1 5 0}$ \\
Testemunha & $14,6 \mathrm{a}^{3}$ & $25,9 \mathrm{a}$ & $22,2 \mathrm{a}$ & $18,8 \mathrm{a}$ & $16,1 \mathrm{a}$ & $24,0 \mathrm{a}$ \\
Raiz 1\% & $8,8 \mathrm{abcd}$ & $6,9 \mathrm{bcd}$ & $7,1 \mathrm{~cd}$ & $8,2 \mathrm{~b}$ & $10,8 \mathrm{~b}$ & $10,0 \mathrm{bc}$ \\
Raiz 2\% & $7,2 \mathrm{bcd}$ & $4,8 \mathrm{~cd}$ & $7,8 \mathrm{bcd}$ & $5,9 \mathrm{bc}$ & $6,9 \mathrm{~b}$ & $3,5 \mathrm{c}$ \\
Raiz 5\% & $4,9 \mathrm{~d}$ & $3,2 \mathrm{~d}$ & $3,9 \mathrm{~d}$ & $3,4 \mathrm{c}$ & $6,8 \mathrm{~b}$ & $3,6 \mathrm{c}$ \\
P. Aérea 1\% & $8,8 \mathrm{abcd}$ & $6,9 \mathrm{bcd}$ & $6,7 \mathrm{~cd}$ & $6,9 \mathrm{bc}$ & $11,3 \mathrm{~b}$ & $12,0 \mathrm{~b}$ \\
P. Aérea 2\% & $6,3 \mathrm{~cd}$ & $8,6 \mathrm{bc}$ & $8,5 \mathrm{bc}$ & $9,5 \mathrm{~b}$ & $8,8 \mathrm{~b}$ & $7,7 \mathrm{bc}$ \\
P. Aérea 5\% & $5,2 \mathrm{~d}$ & $7,6 \mathrm{bcd}$ & $6,5 \mathrm{~cd}$ & $6,1 \mathrm{bc}$ & $7,8 \mathrm{~b}$ & $3,7 \mathrm{c}$ \\
Inflor. 1\% & $11,3 \mathrm{abc}$ & $11,6 \mathrm{~b}$ & $6,2 \mathrm{~cd}$ & $9,2 \mathrm{~b}$ & $9,3 \mathrm{~b}$ & $10,5 \mathrm{bc}$ \\
Inflor. 2\% & $9,0 \mathrm{abcd}$ & $5,7 \mathrm{~cd}$ & $9,6 \mathrm{bc}$ & $10,1 \mathrm{~b}$ & $9,8 \mathrm{~b}$ & $13,0 \mathrm{~b}$ \\
Inflor. 5\% & $12,3 \mathrm{ab}$ & $6,8 \mathrm{bcd}$ & $12,6 \mathrm{~b}$ & $8,1 \mathrm{~b}$ & $10,6 \mathrm{~b}$ & $10,1 \mathrm{bc}$ \\
\hline
\end{tabular}

${ }^{1}$ Dados transformados em $x^{0,5}$ para análise estatística, mas apresentam os valores obtidos; ${ }^{2}$ Dias após a mistura do pó de $C$. iria aos grãos de trigo; ${ }^{3}$ Médias seguidas de letras minúsculas distintas nas colunas diferem entre si pelo teste de Duncan a $5 \%$ de probabilidade dos nas raízes. De acordo com esses autores, o nível desse composto aumentava até o florescimento, diminuía por um período transitório e, em plantas maduras, crescia novamente; só quando a parte aérea se tornava senescente é que o nível de HJ III era reduzido; entretanto, não se observava diminuição do nível de HJ III nos tecidos das raízes (Bede et al., 2001), sugerindo que a flutuação era dinâmica, que ocorria biossíntese ativa, catabolismo e, talvez, transporte desse composto na planta.

Verificou-se, na avaliação realizada logo após o tratamento, que as estruturas de $C$. iria que mais repeliram $S$. oryzae foram aquelas produzidas com pós de raízes e partes aéreas nas concentrações de 2 e 5\% mas a repelência, de acordo com a classificação de Talukder \& Howse (1994), foi média (os valores variaram de 50,7 a 66,4\%) (Tabela 2); aos 30 dias após a mistura dos pós todos os tratamentos repeliram $S$. oryzae sendo a repelência, na maioria dos tratamentos, superior a $70 \%$. De modo geral, nos tratamentos com os pós produzidos com raízes a 5\% a repelência foi superior a $80 \%$ até 150 dias, estrutura esta considerada altamente repelente. A ação repelente é uma propriedade relevante a ser considerada no controle de praga de produtos armazenados, pois quanto maior a repelência menor será a infestação, reduzindo ou suprimindo a postura e, consequentemente, com menor número de insetos emergidos (Coitinho et al., 2006).

Novo et al. (2003) avaliando o efeito de C. iria quando adicionado a pellets de trigo no desenvolvimento de $S$. oryzae, verificaram que a inibição estava diretamente relacionada com a concentração da planta na dieta do inseto devido, provavelmente à presença do HJ III da planta; entretanto, Hong (1975) concluiu, em experimento avaliando hormônios juvenis sintéticos e alguns de seus análogos no desenvolvimento de Ephestia spp. (Lepidoptera: Phycitidae) que, embora a concentração afete a repelência o efeito inseticida varia com a natureza do grão e com o tipo de inseto.

Em relação à repelência dos preparados com as estruturas de $C$. iria verificou-se, para aqueles produzidos com raízes e inflorescência a 5\%, que o período de armazenamento pode ser superior a 150 dias e esses não perderam o efeito residual (Figura 1). Pós produzidos a partir de raízes a 1 e

Tabela 2. Repelência média, em porcentagem, de pós-produzidos a partir de estruturas de C. iria a S. oryzae nas diferentes épocas de avaliação

\begin{tabular}{|c|c|c|c|c|c|c|}
\hline \multirow{3}{*}{ Tratamento } & \multicolumn{6}{|c|}{$\%$ de repelência em relação à testemunha ${ }^{1}$} \\
\hline & \multicolumn{6}{|c|}{ Dias após os tratamentos ${ }^{2}$} \\
\hline & 0 & 30 & 60 & 90 & 120 & 150 \\
\hline Raiz 1\% & $39,7 b^{3}$ & $73,3 \mathrm{a}$ & $68,0 \mathrm{~b}$ & $56,4 \mathrm{c}$ & $32,9 \mathrm{~b}$ & $58,3 \mathrm{c}$ \\
\hline Raiz 2\% & $50,7 \mathrm{a}$ & $81,5 \mathrm{a}$ & $64,8 \mathrm{~b}$ & $68,6 \mathrm{~b}$ & & $85,4 \mathrm{a}$ \\
\hline Raiz 5\% & $66,4 \mathrm{a}$ & 87,6 a & 82,4 a & 81,9 a & $57,7 \mathrm{a}$ & $85,0 \mathrm{a}$ \\
\hline Parte Aérea 1\% & $39,7 \mathrm{~b}$ & $73,3 \mathrm{a}$ & $69,8 \mathrm{~b}$ & $63,3 \mathrm{~b}$ & $29,8 \mathrm{c}$ & $50,0 \mathrm{~cd}$ \\
\hline Parte Aérea 2\% & $56,9 \mathrm{a}$ & $66,8 \mathrm{a}$ & $61,7 \mathrm{~b}$ & $49,5 \mathrm{c}$ & $45,3 \mathrm{~b}$ & $67,9 \mathrm{~b}$ \\
\hline Parte Aérea 5\% & $64,4 \mathrm{a}$ & $70,6 \mathrm{a}$ & $70,7 \mathrm{a}$ & $67,6 \mathrm{~b}$ & $51,6 \mathrm{a}$ & $84,6 \mathrm{a}$ \\
\hline Inflorescência 1\% & $22,6 \mathrm{c}$ & $55,2 \mathrm{~b}$ & $72,1 \mathrm{a}$ & $51,0 \mathrm{c}$ & $42,2 \mathrm{~b}$ & $56,3 \mathrm{c}$ \\
\hline Inflorescência $2 \%$ & $38,3 \mathrm{~b}$ & $78,0 \mathrm{a}$ & $56,7 \mathrm{c}$ & $46,3 \mathrm{c}$ & $39,1 \mathrm{~b}$ & $45,0 \mathrm{~d}$ \\
\hline Inflorescência 5\% & $15,8 \mathrm{c}$ & $73,8 \mathrm{a}$ & $43,2 \mathrm{c}$ & $56,9 \mathrm{c}$ & $34,1 \mathrm{~b}$ & $57,9 \mathrm{c}$ \\
\hline
\end{tabular}

${ }_{1}$ Dados transformados em arcsen $(\mathrm{x} / 100)^{0,5}$ para análise estatística, mas apresentam os valores obtidos; ${ }^{2}$ Dias após a mistura do pó de $C$. iria aos grãos de trigo; ${ }^{3}$ Médias seguidas de letras minúsculas distintas nas colunas diferem entre si pelo teste de Duncan a $5 \%$ de probabilidade 
$5 \%$ apresentaram, respectivamente, a maior e menor repelência aos 46 e 50 dias.

\section{Bioensaio 2: Avaliação da toxicidade e do efeito na progênie de S.oryzae}

A toxicidade dos pós preparados com as estruturas de $C$. iria ao gorgulho nas diferentes épocas de avaliação, é apresentada na Tabela 3. A ação inseticida de C. iria foi dependente da estrutura da planta, da concentração no grão de trigo e do período de infestação. Comparando-se os tratamentos entre si, no dia da aplicação a mortalidade foi baixa, observando-se valores superiores a 15\% apenas nos três tratamentos contendo raiz; aos 30 dias após o tratamento, notou-se nos grãos com $5 \%$ de raiz de $C$. iria, mortalidade superior a 75\%; mas, nas avaliações seguintes esta era inferior a 30\%. De modo geral, até os 90 dias os tratamentos com pós de raízes causaram mais mortalidade que os preparados com as partes aéreas e as inflorescências.

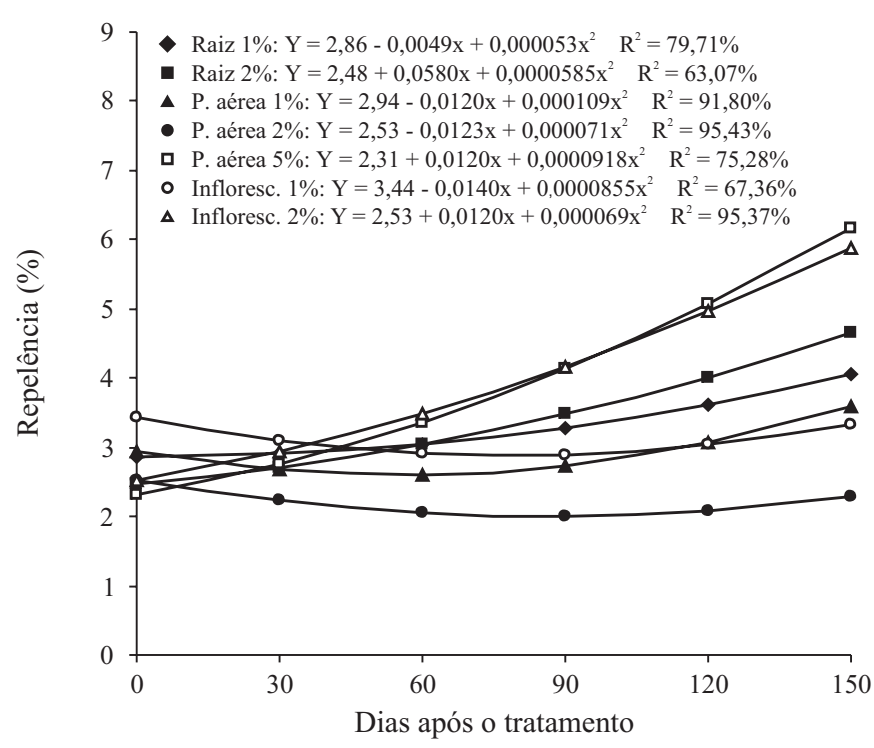

Figura 1. Efeito residual de pós das estruturas de C. iria raiz a 1 e $2 \%$, parte aérea a 1,2 e $5 \%$ e inflorescência a 1 e $2 \%$ quanto à porcentagem de repelência a $S$. oryzae. Dados de \% de repelência transformados em arcsen $(x / 100)^{0,5}$ para análise estatística sendo considerado $5 \%$ de probabilidade de erro

Segundo Edwards et al. (1988), espécies de Sitophilus são menos sensíveis a compostos com hormônio juvenil que outras pragas de grãos armazenados. Sugeriu-se, daí, que as larvas, por estarem dentro dos grãos, ficavam protegidas dos efeitos dos análogos do hormônio juvenil, visto serem estes incapazes de penetrar até os locais onde ocorria o desenvolvimento das larvas e pupas (Bhatnagar-Thomas, 1973); posteriormente, Rowlands (1976) demonstrou que havia três hormônios juvenis que penetravam prontamente nos grãos de trigo e se acumulavam na região da camada de aleurona; como no instar final as larvas estão próximas da camada de aleurona, a insensibilidade de Sitophilus podia ser devida a outros fatores. Este autor observou, ainda, que neste inseto o sistema enzimático era capaz de degradar rapidamente o hormônio juvenil endó-
Tabela 3. Porcentagem média da mortalidade de adultos de $S$. oryzae tratados com pós preparados com as estruturas raiz, parte aérea e inflorescência (infloresc.) de C. iria aos zero, 30, 60, 90 e 120 dias

\begin{tabular}{lccccc}
\hline & \multicolumn{5}{c}{ Porcentagem de mortalidade $^{1}$} \\
\cline { 2 - 6 } Tratamento & \multicolumn{5}{c}{ Dias após 0s tratamentos $^{2}$} \\
\cline { 2 - 6 } & $\mathbf{0}$ & $\mathbf{3 0}$ & $\mathbf{6 0}$ & $\mathbf{9 0}$ & $\mathbf{1 2 0}$ \\
Testemunha & $0,4 \mathrm{c}$ & $1,6 \mathrm{c}$ & $0,0 \mathrm{c}$ & $0,0 \mathrm{c}$ & $0,0 \mathrm{a}$ \\
Raiz 1\% & $17,8 \mathrm{ab}$ & $26,0 \mathrm{~b}$ & $20,2 \mathrm{a}$ & $4,0 \mathrm{bc}$ & $7,3 \mathrm{a}$ \\
Raiz 2\% & $17,4 \mathrm{ab}$ & $1,6 \mathrm{c}$ & $0,4 \mathrm{bc}$ & $7,9 \mathrm{~b}$ & $7,9 \mathrm{a}$ \\
Raiz 5\% & $30,7 \mathrm{a}$ & $77,7 \mathrm{a}$ & $24,2 \mathrm{a}$ & $27,0 \mathrm{a}$ & $8,8 \mathrm{a}$ \\
Parte Aérea 1\% & $9,5 \mathrm{bc}$ & $21,4 \mathrm{~b}$ & $4,8 \mathrm{abc}$ & $1,6 \mathrm{bc}$ & $1,3 \mathrm{a}$ \\
Parte Aérea 2\% & $6,5 \mathrm{bc}$ & $9,5 \mathrm{bc}$ & $10,5 \mathrm{ab}$ & $0,4 \mathrm{bc}$ & $1,6 \mathrm{a}$ \\
Parte Aérea 5\% & $4,8 \mathrm{bc}$ & $14,4 \mathrm{bc}$ & $10,9 \mathrm{ab}$ & $7,9 \mathrm{~b}$ & $7,9 \mathrm{a}$ \\
Infloresc. 1\% & $1,6 \mathrm{c}$ & $7,9 \mathrm{bc}$ & $6,1 \mathrm{abc}$ & $0,0 \mathrm{c}$ & $6,1 \mathrm{a}$ \\
Infloresc. 2\% & $4,8 \mathrm{bc}$ & $6,1 \mathrm{bc}$ & $1,6 \mathrm{bc}$ & $0,0 \mathrm{c}$ & $0,4 \mathrm{a}$ \\
Infloresc. 5\% & $6,5 \mathrm{bc}$ & $13,6 \mathrm{bc}$ & $0,8 \mathrm{bc}$ & $3,7 \mathrm{bc}$ & $1,6 \mathrm{a}$ \\
\hline
\end{tabular}

${ }^{1}$ Dados transformados em arcsen $(\mathrm{x} / 100)^{0,5}$ para análise estatística, mas apresentam os valores obtidos; ${ }^{2}$ Dias após a mistura do pó de $C$. iria aos grãos de trigo; ${ }^{3}$ Médias seguidas de letras minúsculas distintas nas colunas diferem entre si pelo teste de Duncan a $5 \%$ de probabilidade

geno produzido e Sparks \& Hammock (1980) demonstraram que a esterase envolvida no metabolismo do $\mathrm{HJ}$ era importante na regulação do seu nível.

O período residual dos tratamentos com raízes e partes aéreas a $1 \%$ e inflorescências a $2 \%$ foi linearmente reduzido em função do tempo (Figura 2). Quando se empregou raiz a $2 \%$, verificou-se redução na toxicidade estimando-se que o valor mínimo seria obtido aos 63 dias; com o emprego de raiz a 5\%, a toxicidade máxima seria obtida aos 33 dias.

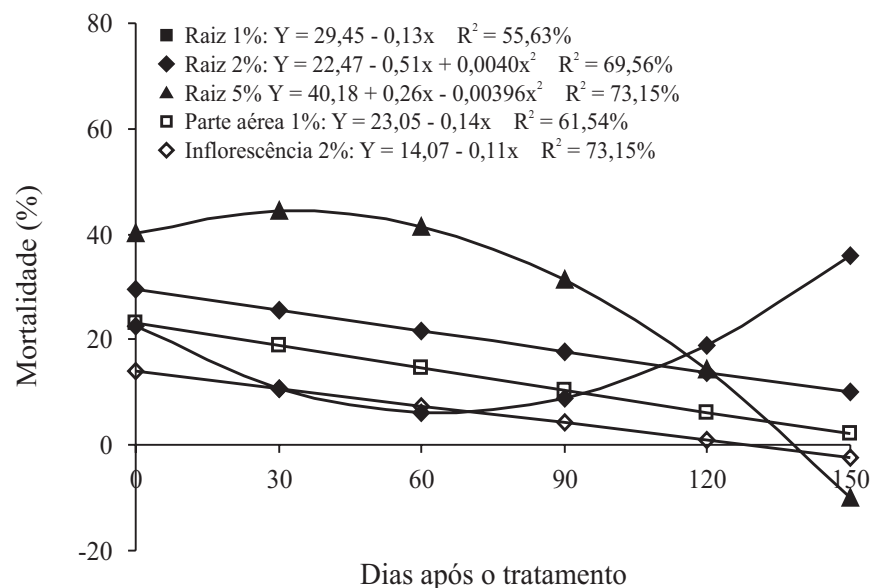

Figura 2. Mortalidade de pós de estruturas de C. iria quanto à mortalidade de $S$. oryzae. Dados de $\%$ de mortalidade transformados em arcsen $(x / 100)^{0,5}$ para análise estatística sendo considerado $5 \%$ de probabilidade de erro

Para progênie houve efeito das estruturas até a avaliação realizada aos 60 dias, observando-se que, aos 30 dias, exceto para os grãos tratados com $2 \%$ de raiz, todos os outros tratamentos reduziram a progênie do gorgulho (Tabela 4). Nos grãos de trigo tratados com $5 \%$ de raízes e de partes aéreas e com 1 e $5 \%$ de pós das inflorescências, a progênie foi reduzida até 60 dias.

Obtiveram-se as maiores reduções de progênie em relação à testemunha (Tabela 5) no tratamento com raiz a 5\%. De modo geral, nesse tratamento a redução da progênie se 
manteve superior a 50\%, mesmo aos 120 dias após a aplicação, revelando bom potencial de controle ao gorgulho; como C. iria possui um hormônio envolvido no controle da metamorfose e na reprodução de insetos contribuiu, provavelmente para a redução da progênie de S. oryzae (Toong et al., 1988).

\section{Bioensaio 3: Avaliação de repelência de extratos aquosos em tiras de papel}

A repelência observada com os extratos produzidos com as estruturas de $C$. iria, nas diferentes concentrações na avaliação realizada logo após os tratamentos, é apresentada na Tabela 6; nesta avaliação os extratos apresentaram somente repelência, com raiz a 0,1 e $0,5 \mathrm{mg} \mathrm{cm}^{-2}$, parte aérea $0,5 \mathrm{mg} \mathrm{cm}^{-2}$ e inflorescência a 0,5 e $1,0 \mathrm{mg} \mathrm{cm}^{-2}$; a maior repelência foi observada com o extrato produzido com raízes a $1,0 \mathrm{mg} \mathrm{cm}^{-2}$ classificado, portanto, de acordo com a classificação de Talukder \& Howse (1994), na classe 4; ressalta-se, entretanto que, embora a repelência com raízes tenha sido classificada na classe 4, está inserida no limite inferior enquanto na avaliação realizada 30 dias após a aplicação não se observou repelência em nenhum dos tratamentos indicando que, além de pouco eficientes, extratos aquosos de $C$. iria não apresentaram efeito residual.

Tabela 4. Efeito das estruturas raiz, parte aérea e inflorescência (infloresc.) de C. iria no número de adultos na progênies de S. oryzae nas diferentes épocas de infestação

\begin{tabular}{lrrrrr}
\hline \multirow{2}{*}{ Tratamentos } & \multicolumn{5}{c}{ Número de adultos na progênie } \\
\cline { 2 - 6 } Testemunha & inicial & 30 dias & 60 dias & 90 dias & 120 dias \\
Raiz 1\% & 77,8 a & $86,6 \mathrm{a}$ & $108,2 \mathrm{ab}$ & $106,6 \mathrm{a}$ & $93,0 \mathrm{a}$ \\
Raiz 2\% & $75,2 \mathrm{a}$ & 56,2 ab & $72,8 \mathrm{c}$ & $76,2 \mathrm{a}$ & $76,8 \mathrm{a}$ \\
Raiz 5\% & $60,4 \mathrm{a}$ & $32,0 \mathrm{~b}$ & $50,2 \mathrm{c}$ & $60,4 \mathrm{a}$ & $67,8 \mathrm{a}$ \\
Parte Aérea 1\% & $59,2 \mathrm{a}$ & $34,8 \mathrm{~b}$ & $72,4 \mathrm{c}$ & $74,4 \mathrm{a}$ & $40,8 \mathrm{a}$ \\
Parte Aérea 2\% & $64,4 \mathrm{a}$ & $32,0 \mathrm{~b}$ & $64,6 \mathrm{c}$ & $84,6 \mathrm{a}$ & $87,8 \mathrm{a}$ \\
Parte Aérea 5\% & $68,0 \mathrm{a}$ & $36,2 \mathrm{~b}$ & $54,8 \mathrm{c}$ & $60,0 \mathrm{a}$ & $80,8 \mathrm{a}$ \\
Infloresc. 1\% & $72,2 \mathrm{a}$ & 40,2 b & $52,2 \mathrm{c}$ & $80,8 \mathrm{a}$ & $84,0 \mathrm{a}$ \\
Infloresc. 2\% & $84,6 \mathrm{a}$ & $36,0 \mathrm{~b}$ & $83,8 \mathrm{bc}$ & $76,2 \mathrm{a}$ & $96,4 \mathrm{a}$ \\
Infloresc. 5\% & $121,4 \mathrm{a}$ & $36,0 \mathrm{~b}$ & $73,2 \mathrm{c}$ & $70,6 \mathrm{a}$ & $111,6 \mathrm{a}$ \\
\hline
\end{tabular}

${ }^{1}$ Dados transformados em $(x+1)^{0,5}$ para análise estatística, mas apresentam os valores obtidos; ${ }^{2}$ Médias seguidas de letras minúsculas distintas nas colunas diferem entre si pelo teste de Duncan a $5 \%$ de probabilidade

Tabela 5. Redução percentual na progênie de $S$. oryzae em relação à testemunha em cada época de infestação. Campinas, SP, 2003

\begin{tabular}{|c|c|c|c|c|c|}
\hline \multirow{3}{*}{ Tratamentos } & \multicolumn{5}{|c|}{$\%$ de redução em relação à testemunha ${ }^{1}$} \\
\hline & \multicolumn{5}{|c|}{ Dias após os tratamentos ${ }^{2}$} \\
\hline & inicial & 30 & 60 & 90 & 120 \\
\hline Raiz 1\% & $0,0 \mathrm{~b}^{3}$ & $61,0 \mathrm{a}$ & $32,7 \mathrm{c}$ & $28,5 \mathrm{~b}$ & $17,4 \mathrm{bc}$ \\
\hline Raiz 2\% & $3,3 \mathrm{~b}$ & $35,1 \mathrm{~b}$ & $0,0 \mathrm{e}$ & $24,6 \mathrm{~b}$ & $27,1 \mathrm{~b}$ \\
\hline Raiz 5\% & $22,4 \mathrm{a}$ & $63,1 \mathrm{a}$ & 53,6 a & $40,3 \mathrm{a}$ & $56,1 \mathrm{a}$ \\
\hline Parte Aérea 1\% & $23,9 \mathrm{a}$ & $59,8 \mathrm{a}$ & $33,1 \mathrm{c}$ & $30,2 \mathrm{a}$ & $37,9 \mathrm{~b}$ \\
\hline Parte Aérea 2\% & $17,2 \mathrm{a}$ & $63,1 \mathrm{a}$ & $40,3 \mathrm{~b}$ & 20,6 a & $5,6 \mathrm{~d}$ \\
\hline Parte Aérea 5\% & $12,6 \mathrm{ab}$ & $58,2 a b$ & $49,4 \mathrm{~b}$ & $43,7 \mathrm{a}$ & $13,1 \mathrm{c}$ \\
\hline Inflorescência 1\% & $7,2 \mathrm{ab}$ & $53,6 a b$ & $51,8 \mathrm{a}$ & $24,2 \mathrm{~b}$ & $9,7 \mathrm{~d}$ \\
\hline Inflorescência 2\% & $0,0 \mathrm{~b}$ & $58,4 a b$ & $22,6 \mathrm{~d}$ & $28,5 \mathrm{~b}$ & $0,0 \mathrm{~d}$ \\
\hline Inflorescência 5\% & $0,0 \mathrm{~b}$ & $58,4 a b$ & $32,4 \mathrm{c}$ & $33,8 \mathrm{a}$ & $0,0 \mathrm{~d}$ \\
\hline
\end{tabular}

${ }_{1}$ Dados transformados em arcsen $(x / 100)^{0,5}$ para análise estatística, mas apresentam os valores obtidos; ${ }^{2}$ Dias após a mistura do pó de $C$. iria aos grãos de trigo; ${ }^{3}$ Médias seguidas de letras minúsculas distintas nas colunas diferem entre si pelo teste de Duncan a $5 \%$ de probabilidade
Tabela 6. Repelência média, em porcentagem, de extratos aquosos produzidos a partir de pós de raízes $(\mathrm{R})$, parte aérea $(\mathrm{PA})$ e inflorescências (I) aplicados em papel sulfite a $S$. oryzae na avaliação realizada logo após a contaminação e sua classificação pela escala de Taludker \& Howse (1994)

\begin{tabular}{|c|c|c|c|c|c|}
\hline \multicolumn{2}{|r|}{ Tratamento } & \multirow{2}{*}{$\begin{array}{r}\text { Test. } \\
51,18\end{array}$} & \multirow{2}{*}{$\begin{array}{l}\text { Trat. } \\
48,82\end{array}$} & \multirow{2}{*}{$\begin{array}{l}\text { Teste } \mathbf{t}^{1} \\
0,46\end{array}$} & \multirow{2}{*}{$\begin{array}{c}\text { Classificação } \\
\text { Talukder \& Howse } \\
\text { (1994) } \\
3\end{array}$} \\
\hline $\mathrm{R}$ & $0,1 \mathrm{mg} \mathrm{cm}^{-2}$ & & & & \\
\hline $\mathrm{R}$ & $0,5 \mathrm{mg} \mathrm{cm}^{-2}$ & 53,75 & 46,25 & $0,0276^{*}$ & 3 \\
\hline $\mathrm{R}$ & $1,0 \mathrm{mg} \mathrm{cm}^{-2}$ & 38,50 & 61,5 & $0,0002^{\star *}$ & 4 \\
\hline PA & $0,1 \mathrm{mg} \mathrm{cm}^{-2}$ & 42,99 & 57,01 & $0,0009^{* *}$ & 3 \\
\hline PA & $0,5 \mathrm{mg} \mathrm{cm}^{-2}$ & 52,67 & 47,33 & 0,26 & 3 \\
\hline $\mathrm{PA}$ & $1,0 \mathrm{mg} \mathrm{cm}^{-2}$ & 47,92 & 52,08 & 0,3 & 3 \\
\hline I & $0,1 \mathrm{mg} \mathrm{cm}^{-2}$ & 49,67 & 50,33 & 0,86 & 3 \\
\hline I & $0,5 \mathrm{mg} \mathrm{cm}^{-2}$ & 43,52 & 56,48 & $0,0319 *$ & 3 \\
\hline I & $1,0 \mathrm{mg} \mathrm{cm}^{-2}$ & 53,83 & 46,17 & $0,0253^{*}$ & 3 \\
\hline
\end{tabular}

${ }^{1}$ Significância do teste t: * significativo a $5 \%$ de probabilidade; ${ }^{* *}$ significativo a $1 \%$ de probabilidade

\section{CONCLUSÕES}

1. Os pós produzidos com qualquer uma das estruturas de $C$. iria repelem $S$. oryzea.

2. A repelência é maior e menor quando usados, respectivamente $5 \%$ de raízes e qualquer concentração da inflorescência.

3. A repelência de raízes a $5 \%$ para $S$. oryzae se mantém por períodos superiores a 150 dias e a progênie é reduzida até 60 dias.

4. A mortalidade e a redução na progênie são maiores quando empregados pós produzidos a partir de raízes de C. iria.

5. Quando empregados extratos aquosos das estruturas de C. iria, a maior repelência é obtida com raízes a $1,0 \mathrm{mg} \mathrm{cm}^{-2}$.

6. O tratamento de grãos com pós de $C$. iria é mais eficiente no controle de $S$. oryzae que os extratos aplicados em papel.

\section{LITERATURA CITADA}

Abbott, W. S. A method of computing the effectiveness of an insecticide. Journal of Economic Entomology, v.18, p.265-266, 1925.

Bede, J. C.; Teal, P. E. A.; Goodman, W. G.; Tobe, S. S. Biosynthesis pathway of insect juvenile hormone III in cell suspension cultures of the sedge Cyperus iria. Plant Physiology, v.127, p.584-593, 2001.

Bede, J. C.; Tobe, S. S. Activity of insect juvenile hormone III: Seed germination and seedling growth studies. Chemoecology, v.10, p.89-97, 2000.

Bhatnagar-Thomas, P. L. Control of insect pests of stored grain using a juvenile hormone analogue. Journal of Economic Entomology, v.66, p.277-278, 1973.

Boff, M. I. C.; Bogo, A.; Boff, P. Atividade inseticida de extratos de pimenta-do-reino, Piper nigrum (Piperaceae) sobre a progênie da traça-dos-cereais, Sitotroga cerealella (Lepidoptera: Gelechiidae). Revista Brasileira de Armazenamento, v.30, n.2, p.111-116, 2005. 
Boff, M. I. C.; Sartori, D. V.; Bogo, A. Efeito de extratos de Piper nigrum L. sobre o caruncho-do-feijoeiro, Acanthoscelides obtectus (Say). Revista Brasileira de Armazenamento, v.31, n.1, p.17-22, 2006.

Bogorni, P. C.;. Vendramim, J. D. Bioatividade de extratos aquosos de Trichilia spp. sobre Spodoptera frugiperda (J. E. Smith) (Lepidoptera: Noctuidae) em milho. Neotropical Entomology, v.32, n.4, p.665-669, 2003.

Coitinho, R. L. B. C.; Oliveira, J. V.; Gondim Júnior, M. G. C.; Câmara, C. A. G. Atividade inseticida de óleos vegetais sobre Sitophilus zeamais Mots (Coleoptera: Curculionidae) em milho armazenado. Revista Caatinga, v.19, n.2, p.176-182, 2006.

Dales, M. J. A. Review of plant materials used for controlling insect pests of stored products. Chatam: Natural Resources Institute. 1996. 84p.

Edwards, J. P.; Short, J. E.; Abraham, L. Large-scale evolution of the insect juvenile hormone analogue fenoxycarb as a longterm protection of stored wheat. Journal of Stored Product Research, v.27, n.1, p.31-39, 1991.

Edwards, J. P.; Short, J. E.; Rowlands, D. G. Metabolism and insect juvenile hormone activity of methoprene and three major metabolites in Tribolium castaneum (Herbst) pupae. Journal of Stored Product Research, v.24, n.3, p.165-172, 1988.

Gallo, D.; Nakano, O.; Silveira Neto, S.; Carvalho, R. P. L.; Batista, G. C.; Berti Filho, E.; Parra, J. R. P.; Zucchi, R. A.; Alves, S. B.; Vendramin, J. D.; Marchini, L. C., Lopes, J. R. S., Omoto, C. Entomologia agrícola. Piracicaba: FEALQ, 2002. 920p.

Gomes, F. P.; Garcia, C. H. Estatística aplicada a experimentos agronômicos e florestais: exposição com exemplos e orientações para uso de aplicativos. Piracicaba: FEALQ, 2002. 309p.

Hong, T. K. Effects of a synthetic juvenile hormone and some analogues on Ephestia spp. (Lepidoptera: Phycitidae). Annals of Applied Biology, v.80, p.137-145, 1975.

Lorenzi, H. Manual de identificação e controle de plantas daninhas: plantio direto e convencional. $6^{\text {a }}$. ed. Nova Odessa: Instituto Plantarum, 2006. 339p.
Meneses, C. R.; Garcia de la Osa, J. Principal weed hosts of Hydrellia sp. in the southern rice-growing zone of Sancti Spiritus. Centro Agrícola, v.15, n.3, p.90-92, 1988.

Metcalf, C. L.; Flint, W. P. Destructive and useful insects: their habit and control. London: McGrawHill, 1962. 1087p.

Mohan, S.; Fields, P.G. A simple technique to assess compounds that are repellent or attractive to stored-products insects. Journal of Stored Products Research, v.38, p.23-31, 2002.

Novo, J. P. S.; Novo, M. C. S. S.; Pilli, L. H.; Capps, A. L. A. P. Efeito de Cyperus iria L. (Cyperaceae) no desenvolvimento de Sitophylus oryzae (L., 1763) (Coleoptera: Curculionidae) em pellets de trigo (Triticum aestivum L.) - Poaceae. In: Reunião Anual do Instituto Biológico, 16, 2003, São Paulo. Resumos... São Paulo: Instituto Biológico, 2003. p.364-368, 2003. Suplemento

Pungitore, C. R.; García, M.; Gianello, J. C.; Sosa, M. E.; Tonn, C. E. Insecticidal and antifeedant effects of Junellia aspera (Verbenaceae) triterpenes and derivatives on Sitophilus oryzae (Coleoptera: Curculionidae). Journal of Stored Products Research, v.41, p.434-443, 2005.

Rowlands, D. G. The uptake and metabolism by stored wheat grains of an insect juvenile hormone and two insect hormone mimics. Journal of Stored Products Research, v.12, n.1, p.35-41, 1976.

Sparks, T. C.; Hammock, B. D. Comparative inhibition of the juvenile hormone estereases from Trichoplisia ni, Musca domestica and Tenebrio molitor. Pesticide, Biochemistry and Physiology, v.14, p.290-301, 1980.

Steel, R. G. D.; Torre, J. H. Principles and procedures of statistics: A biometrical approach. New York: McGrow Hill, 1981. 633p.

Su, H. C. F. Laboratory study on effects of Anethum graveolens seeds on four species of stored-product insects. Journal of Economic Entomology, v.78, n.2, p.451-453, 1985.

Talukder, F. A.; Howse, P. E. Repellent, toxic and food protectant effects of pithraj, Aphanamixis polystachya extracts against pulse beetle, Callosobruchus chinensis in storage. Journal of Chemical Ecology, v.20, p.899-908, 1994.

Toong, Y. C., Schooley, D. A.; Baker, F. C. Isolation of insect juvenile hormone III from a plant. Nature, v.333, p.170-171, 1988. 